\title{
Celulares: contaminación, manejo de residuos y alternativas de cambio
}

\section{Mobile Phones: Pollution, Waste Management, and Alternatives for Change}

\author{
Ángela Gabriela Cubillos Rodríguez, Miguel Alfredo Correa Córdoba, \\ Diana Sofía Vidal Gualteros', Sergio David Díaz Marín 9
}

\section{Resumen}

La constante innovación de celulares ha generado un consumo masivo de los mismos, trayendo consigo malos hábitos de desecho de los dispositivos que ya cumplieron su vida útil. En el presente estudio, se analizaron las actitudes y comportamientos de las personas en cuanto al desecho de los teléfonos móviles con la finalidad de saber las causas que llevan a la decisión de deshacerse de su celular y de qué manera lo hacen. Además, se conoció qué tan dispuestas están las personas a ser parte de una alternativa que ayude al medio ambiente, la cual estaba enfocada en la adquisición de celulares eco-amigables de un mayor costo. Para conseguirlo, se realizó una encuesta a 159 personas vinculadas a la Fundación Universitaria del Área Andina, las cuales además de brindar la información necesitada, permitieron comparar datos con otros estudios relacionados con la materia; asimismo, se evidenció el lamentable hecho de que las personas tienen conocimiento del daño ambiental causado por el desecho indebido de los celulares, pero no acatan medidas que ayuden a mitigarlo. Por otra parte, como aporte relevante, se observó que las personas sí estarían dispuestas a ser

6 Estudiante, Fundación Universitaria del Área Andina, Facultad de Diseño, Comunicación y Bellas Artes, sede Bogotá, Técnico Profesional en Gastronomía y Culinaria. Correo electrónico: acubillos13daestudiantes.areandina.edu.co

7 Estudiante, Fundación Universitaria del Área Andina, Facultad de Diseño, Comunicación y Bellas Artes, sede Bogotá, Técnico Profesional en Gastronomía y Culinaria. Correo electrónico: mcorrea59destudiantes.areandina.edu.co

8 Estudiante, Fundación Universitaria del Área Andina, Facultad de Diseño, Comunicación y Bellas Artes, sede Bogotá, Técnico Profesional en Gastronomía y Culinaria. Correo electrónico: dvidal4Aestudiantes.areandina.edu.co

9 Estudiante, Fundación Universitaria del Área Andina, Facultad de Diseño, Comunicación y Bellas Artes, sede Bogotá, Técnico Profesional en Gastronomía y Culinaria. Correo electrónico: sdiaz94destudiantes.areandina.edu.co 
parte de alternativas que contribuyan al mejoramiento de la calidad ambiental, ya sea reciclando, conociendo los puntos de recolección y desecho adecuado de celulares, o adquiriendo un dispositivo móvil ecoamigable.

Palabras clave: alternativa eco-amigable, daño ambiental, desecho tecnológico.

\section{Abstract}

The constant innovation of mobile phones has boosted their massive consumption, bringing about improper disposal of devices that have already reached their useful life. The study analyzes the attitudes and behaviors of people regarding the disposal of mobile phones to determine the causes for getting rid of them and how they do it. Besides, it reveals how willing people are to be part of an alternative that helps the environment: acquiring eco-friendly mobile phones at a higher cost. For this, surveys were conducted with 159 people from Fundación Universitaria del Área Andina, which provided the information needed and allowed comparing data with other studies on the matter. Unfortunately, we noted that individuals are aware of the environmental damage caused by the improper disposal of mobile phones but do not take measures to mitigate it. Moreover, as a relevant contribution, we observed that respondents would be willing to seek alternatives that improve environmental quality, either by recycling, taking old mobiles to the appropriate collection and disposal points, or acquiring an eco-friendly device.

Keywords: Technological waste, environmental damage, eco-friendly alternative.

\section{Introducción}

¿Conoce usted el impacto ambiental que puede generar un celular en la basura? Probablemente la mayoría de las personas dirá: no, y aunque pueda verse como algo insignificante, la realidad es que con el pasar de los minutos se generan constantes desechos de dispositivos móviles en el mundo. Sea cual sean las causas de su desuso, botarlos sin tener conocimiento del adecuado manejo y reciclaje que requieren puede acarrear diversos daños al medio ambiente. Solo basta imaginar la gran pila de basura electrónica acumulada desde varias dé- 
cadas y expuesta a la humedad y la luz solar, penetrando sustancias tóxicas en el suelo que contribuyen al daño ambiental.

Para este trabajo se buscó determinar los diferentes medios, aspectos $\mathrm{y}$ factores influyentes y responsables que causa la contaminación ambiental de la basura electrónica, tanto en materiales y sustancias tóxicas, derivadas del desecho tecnológico de dispositivos móviles; asimismo, conocer cuál es el nivel de conocimiento, percepción y responsabilidad ambiental que las personas tienen de la promoción de tecnologías verdes.

Con el fin de identificar los materiales tóxicos que se encuentran en los celulares, Singh et al. (2019) evalúan por medio de un estudio, expuesto en su artículo "Tendencias de toxicidad en los desechos electrónicos: un análisis comparativo de metales en teléfonos móviles desechados", que los celulares deben ser clasificados para establecer la cantidad de agentes contaminantes, los cuales están presentes en la composición física del teléfono (excluyendo la batería y la carcasa de estos). Vemos como la clasificación de agentes contaminantes desemboca en 2 grandes categorías: 1) teléfonos básicos y 2) teléfonos inteligentes, con la particularidad de que estos habían sido fabricados entre el 2001 y el
2015. Para llegar a esta clasificación, se utilizan 20 teléfonos, $50 \%$ encasillados como básicos y $50 \%$ como inteligentes, los cuales presentan similares índices de agentes tóxicos, tales como el berilio, plomo, arsénico, mercurio, cobalto, vanadio, entre muchos otros elementos. El fin de estas categorías resultan de la necesidad de puntualizar los impactos potenciales de la toxicidad (los cuales recaen en la salud humana y en el deterioro ecológico), por medio de la comparación de los valores de cada uno de los elementos tóxicos presentes en ambas categorías.

En el estudio realizado por Pascuas et al. (2018), titulado "Residuos de celulares y tabletas: incidencia del nivel de escolaridad en las actitudes y percepciones frente a su manejo", se dan a conocer los hábitos de consumo y las percepciones de los ciudadanos en cuanto a la vida útil de un celular o tablet, y su incidencia en el medio ambiente, estudio realizado a estudiantes de primaria y secundaria de las instituciones educativas de Florencia (Caquetá), así como a estudiantes y egresados pertenecientes a la Universidad de la Amazonía, cuya muestra fue de 450 personas al azar. Dentro de los ítems relacionados a temas ambientales y contaminación producida por celulares, se evidencia que el 
$69 \%$ de la población encuestada guarda su dispositivo y, luego de un tiempo, bota los celulares en lugares no adecuados. Respecto al conocimiento sobre de sitios especializados donde se pueden desechar los aparatos que ya no sirven, es posible identificar que el $70 \%$ de la población en estudio está desinformada. Por otro lado, el $97 \%$ es consciente sobre la importancia del cuidado del medio ambiente, pero que al no estar informada en temas relacionados con el buen manejo de los residuos de teléfonos y $t a$ blets acaban por actuar de manera contraria. Solo el 5,5\% de los encuestados muestra una postura de conocimiento y práctica del buen manejo de los aparatos móviles cuando estos cumplen su vida útil. Teniendo en cuenta lo anterior, esta investigación logra mostrar la necesidad de crear estrategias educativas que permitan, además de concientizar, formar a las personas para que hagan buen uso de los desechos electrónicos y así contribuir en la solución de problemáticas ambientales en Colombia y el mundo.

1 siguiente estudio analizado, fue realizado por Ruiz Galeano \& Bautista Rodríguez (2021), llamado “An integrated method of environmental analysis and system dynamics for management of mobile phone waste in Colombia", y tiene como objetivo identificar los aspec- tos relevantes que influyen en la creciente generación de residuos electrónicos a partir del desecho inadecuado de los teléfonos móviles, como también el impacto ambiental que producen, destacando que la cantidad de $\mathrm{CO}_{2}$ que producen estos dispositivos depende por completo de su vida útil. Con el fin de cuantificar e identificar la etapa de uso de los dispositivos móviles, en el 2018, se realizó una encuesta a 358 personas de 24 ciudades colombianas elegidas al azar, usuarias de telefonía móvil. Dicha encuesta se llevó a cabo por medio de redes sociales, teniendo aspectos, tales como rangos e ingresos económicos, recopilando datos con respecto al uso que le destinaron los usuarios a su teléfono móvil cuando este cumplió su ciclo de vida útil. En esta encuesta se observó la variabilidad en las respuestas de los usuarios, puesto que unos afirman haberle dado un segundo ciclo de vida a su teléfono al reciclarlo y así reducir su impacto ambiental. Otros, por el contrario, no recuerdan dónde lo dejaron o en algunos casos lo botaron a la basura porque no tenían conocimiento de compañías que se encargaran de este tipo de desechos. En conclusión, dichos datos fueron destinados a un proyecto investigativo para estimar la generación de residuos de teléfonos móviles en Colombia y su finalidad dada por la población en ge- 
neral, y así lograr calcular los índices de $\mathrm{CO}_{2}$ del dispositivo en uso y el aumento de los desechos que se podrían producir debido a la eliminación de los mismos.

Nnorom et al. (2009) realiza una investigación sobre el deterioro de la calidad ambiental debido al desecho inadecuado de los teléfonos móviles, en la cual, se realiza una encuesta a 1000 personas de dos zonas distintas de Nigeria: Okigwe (zona semiurbana de rápido desarrollo) y Isuikwuato (zona predominante rural), cercanas a la Universidad Estatal de Rabia. Para la encuesta se tuvieron en cuenta los siguientes factores: poblaciones educadas y no educadas, con diferentes tipos de ingreso económico y con uso frecuente de teléfono celular. Partiendo de los resultados de esta encuesta, se observó que el 64,63\% de la población es consciente del deterioro ambiental que ha tenido Nigeria en los últimos 10 años, por tal motivo, el 74,02 $\%$ de la misma población encuestada está dispuesta a pagar un excedente de $20 \%$ de prima por un teléfono verde. La mayoría de los encuestados evidenció una postura positiva hacia un cambio, el cual se basa en entregar o dejar los dispositivos móviles que ya no utilizan en un sitio adecuado de reciclaje y así poder contribuir a la disminución del deterioro ambiental.

\section{Impacto del comportamiento humano en el medio ambiente}

Deen (2019) describe como la humanidad depende del entorno ambiental y los recursos del mismo. La creciente competitividad ha incurrido en la explotación de los recursos naturales contribuyendo en la degradación del medio ambiente, evidencia no solo de los negativos e irremediables impactos, sino también cambios a nivel social. El hecho de que la innovación tecnológica sea cada año más asequible tiene un costo devastador, ya que cada año se acumulan más de 50 millones de toneladas de desechos electrónicos en todo el planeta, lo cual es mayor que la chatarra anual generada por las aerolíneas en todo el mundo. La Universidad de las Naciones Unidas pronostica que, de no haber algún cambio, estos desechos se triplicarán para el 2050. Los residuos electrónicos constituyen actualmente en la basura de más rápido crecimiento en el mundo y, por lo tanto, una de las que más porcentaje de contaminación aporta al medio ambiente.

\section{Desechos y contaminación}

Dang \& Zhang (2021) sostienen que los dispositivos de alta tecnología influyen cada vez más en la sociedad 
y, con el tiempo, han cobrado un papel muy importante en la sociedad moderna, debido a su constante innovación, mientras que su vida útil se vuelve cada vez más corta debido a factores como obsolescencia física, actualizaciones que requieren últimas versiones, o la interrupción del soporte para modelos más antiguos. Por lo tanto, los residuos generados por dichos dispositivos han crecido en todo el mundo a razón de 44,7 millones de toneladas métricas anuales, de los cuales se documentó que solo al $20 \%$ se del da un trato adecuado (recolectando, clasificando y reciclando). En resumen, más de 36 millones de toneladas se arrojan inescrupulosamente a los desechos residuales, se vierten, comercializan o reciclan en condiciones deficientes, sumando así a una desproporcionada contaminación ambiental. Hace muchos años, la basura residual de los países industrializados era llevada a los países en vía de desarrollo, debido a costos de transporte más baratos y una legislación débil, esto ocasiona que cada vez más hubiese un considerable aumento de contaminación en dichos países, los cuales hoy en día presentan daños considerados por muchos como irreparables y catastróficos.

\section{Contaminación en la era tecnológica}

Zornosa Prieto (2009) analiza los riesgos emergentes de la era tecnológica, enfocados especialmente en los daños al medio ambiente, como también en los daños ecológicos en general. El evento desencadenado por una contaminación paulatina de diversos dispositivos móviles, evidentemente, tienen consecuencias, como emanar sustancias tóxicas que luego son absorbidas por el entorno. Es casi incalculable estimar la magnitud del siniestro, debido al constante crecimiento de compra e indebido desecho de dispositivos móviles que generan contaminación. Estos datos son indispensables para un cambio en la sociedad y entorno en el que la misma se desplaza, ya que se evidencia con más claridad las consecuencias de no darles un adecuado manejo, a corto, mediano y largo plazo. De la misma manera, es difícil evaluar con exactitud cuántos productos electrónicos se fabrican al año; sin embargo, considerando únicamente que los dispositivos conectados a Internet totalizan una suma mucho mayor que la de humanos, cuya población mundial es de unos 7700 millones, el número de dispositivos es casi irreal. 


\section{Desechos tecnológicos}

Mihai et al. (2019) definen los desechos tecnológicos, o también llamados E-waste, como una corriente de residuos emergentes del desarrollo y consumo de aparatos electrónicos que emergen a nivel mundial. Los residuos pueden estar presentes como "basura", pero también acumulados en los hogares. En la actualidad, estos desechos no presentan un manejo adecuado, pues, aunque hay un protocolo para su eliminación por parte de las empresas creadoras de estos dispositivos, sus esfuerzos no son suficientes para tener control de los residuos generados en algunas partes del mundo.

\section{Generalidades de la tecnología del dispositivo móvil}

Christensen y Foss (2021) explican que un dispositivo móvil es un aparato electrónico inteligente de fácil manejo que ofrece flexibilidad e intercambio de información entre el mismo y uno semejante, teniendo como fin facilitar distintas tareas que lo requieran. Entre los aparatos que integran este grupo de dispositivos móviles inteligentes — también llamados smart devices-, se encuentran los televisores, computadores portátiles, celulares, relojes y tablets.
El teléfono celular es el dispositivo electrónico más utilizado en el mundo, lo que se puede explicar con el consumismo que genera este. Su creación facilita muchas actividades que realizan las personas, principalmente por la funcionalidad de sus herramientas, pero no significa que no acarree problemas consigo.

Coyne et al. (2019) deja en evidencia las dificultades que se pueden desarrollar a causa del uso de los teléfonos móviles, en cuanto al aspecto psicológico de la persona, desde la etapa de adolescencia de un individuo hasta su etapa de madurez se pueden evidenciar algunos trastornos sociales, depresión, ansiedad, adicción a los aparatos tecnológicos, entre otros factores que pueden llegar a generan un perjuicio a los usuarios de estas tecnologías.

\section{Efectos de la tecnología por su composición: toxicidad}

Como variable para añadir a las complicaciones que pueden proceder del uso de un teléfono móvil es la toxicidad, puesto que la composición de estos necesita de elementos que generan no solo una contaminación para el medio ambiente, sino que suponen un riesgo para la salud humana. 
Los niveles de toxicidad varían según el tipo de teléfono móvil, como lo expone Singh et al. (2019) en su estudio, demostrando que el teléfono posee sustancias que resultan nocivas, pero son indispensables para la fabricación y funcionamiento de los celulares. Teniendo en cuenta que los componentes varían en tipo y volumen, pueden ser clasificados por su cantidad y toxicidad. El elemento con mayor contenido y mayor toxicidad es el cobre $(\mathrm{Cu})$, variando por el tipo de teléfono (bien sea inteligente o básico), estableciendo niveles críticos de ecotoxicidad que oscilan entre los 45,818 - 77,938 PAF m ${ }^{3} / \mathrm{kg}$ (siendo esto resultado de una medida de impacto por cobre contenido en un teléfono producido en grandes cantidades y que es desechado, y ocupa un espacio dedicado a los desechos).

Partiendo de los resultados de la búsqueda de elementos contaminantes que se encuentran en la composición física de los teléfonos celulares, se observa que la producción masiva de estos aparatos electrónicos acarrea consigo una agravante contaminación en masa. A pesar de que las cantidades de estas sustancias son de algunos microgramos por aparato, la suma de todas los agentes contaminantes y su impacto ambiental resultan en la creación de una escala especial para medir los mismos (PAF=medida de impacto). El elemento con mayor índice de ecotoxicidad presente en los teléfonos celulares (sea o no inteligente) es el cobre, con índices PAF relacionados a un estado crítico, lo que significa un riesgo para el medio ambiente. Por otro lado, se evidencian factores que influyen en la contaminación, donde la economía prima por encima de las demás, viendo casos en el que cierto tipo de materiales que son utilizados en la fabricación de esta tecnología resultan rentables para la producción y eficientes para el funcionamiento de estos. La innovación trae consigo el desecho masivo de los teléfonos móviles, donde nuevas funciones o características estéticas convierten en obsoletos a la tecnología anterior, a lo último en el mercado: por esto, la búsqueda de tecnología verde puede ser un pequeño paso en la lucha contra la contaminación tecnológica provocada por el desecho de los aparatos electrónicos.

\section{Alternativa ambiental: tecnología verde}

Fujii y Managi (2019) enuncia que la tecnología verde es crucial para lograr la conservación de energía, la reducción de emisores y el crecimiento económico, también que es importante para la creación de una sociedad sostenible al promover el 
cuidado del medio ambiente y el desarrollo económico.

\section{Eco-celulares}

Athena Information Solutions Pvt. Ltd. en su publicación, "What 's Next: en eco friendly cell phones made using green materials" (EcoFriend, 2011), expone como los dispositivos móviles ecológicos son la última innovación de los fabricantes de teléfonos móviles. Estos teléfonos celulares están hechos de materiales biodegradables a los cuales se les hace un estudio específico de resistencia, para poder sacarlo a la venta. Los primeros teléfonos móviles ecológicos que se crearon no generaron un buen impacto para la sociedad, pero se logró un gran avance, ya que estos teléfonos mostraron un abrebocas de lo que se viene para el futuro. Actualmente, se siguen realizando estudios para la generación de un celular que sea al menos un $60 \%$ amigable con el medio ambiente y que sorprenda a la sociedad con su rendimiento.

En la actualidad, se sigue batallando por la creación de un dispositivo que reúna las expectativas de rendimiento que la sociedad espera, obviamente, este dispositivo tiene que ser por mínimo un $60 \%$ ecoamigable, lo que se busca a futuro es que sea un $100 \%$, pero en un mundo donde solo importa las tendencias, es complejo.

\section{Resultados}

La metodología de esta investigación se desarrolló por medio de exploración bibliográfica y de consulta por medio de encuestas de manera virtual, a las cuales se les suministró un formulario conformado por 14 preguntas, distribuidas en 2 secciones: la primera sección formada por 4 preguntas caracterizadoras y la segunda formada por 10 preguntas especializadas, las cuales tenían como fin conocer su percepción respecto a la problemática tratada en este artículo. La población encuestada fue de 159 personas pertenecientes a la Fundación Universitaria del Área Andina, de los cuales el $67,3 \%$ son hombres y el $32,7 \%$ son mujeres. Los rangos de edades de los encuestados oscilan entre 18-29 años correspondientes al 73,6 \%, 30-45 años corresponden al 18,9 \%, 15-17 años corresponden al $5 \%$ y entre los $46-55$ años que son el 2,5\%. Respecto al último nivel de escolaridad obtenido, se evidencia que el $78 \%$ corresponde a básica primaria y el $22 \%$ a educación media. Finalmente, los resultados correspondientes a la ocupación de los encuestados, se obtuvo que el 85,5 $\%$ son estudiantes universitarios y el 14,5 $\%$ son profesionales.

Con respecto a las actitudes y percepciones que tienen las personas frente al desecho de celulares y su incidencia 
en el medio ambiente se observa las respuestas en la figura 1:

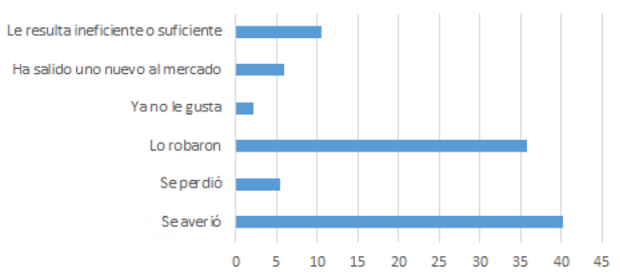

Figura 1. Razones de cambio de celular. Fuente: elaboración propia.

En la figura 1, se evidencia la respuesta a la pregunta: ¿por qué razón o razones ha cambiado de celular? El porcentaje de respuestas obtenido fue el siguiente: Se averió con un 40,2 \%; Lo robaron con $35,8 \%$; seguido se encuentran: Ineficiente o insuficiente con un 10,5\%; Se perdió con un 5,4\%; Ya no le gusta con un 10,5\% y, por último, Ha salido uno nuevo al mercado con un $5,9 \%$.

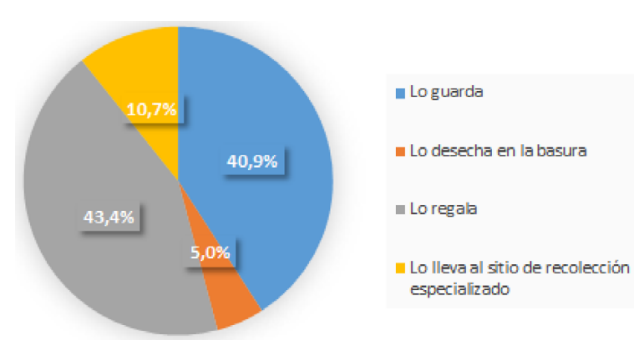

Figura 2. Lo que pasa con los celulares que no se usan.

Fuente: elaboración propia.
En la figura 2, se reflejan las respuestas a la pregunta: ¿qué hace con el celular que ya no usa? A lo cual, un 43,4 $\%$ respondió que Lo regala; posteriormente, se encuentra un 40,9\% que contestó Lo guarda; por otro lado, un 10,7\% evidencia que Lo llevan a un sitio de recolección especializado, y tan solo un 5 $\%$ señala que Lo desechan en la basura.

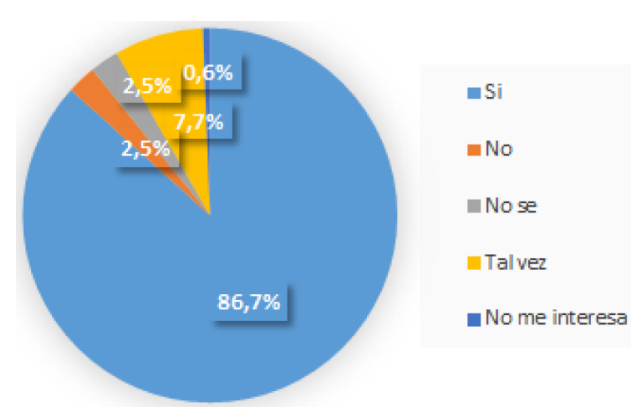

Figura 3. Percepción del daño que se produce al desechar un celular a la basura.

Fuente: elaboración propia.

En la figura 3, se encuentra respuesta a la pregunta: ¿cree usted que desechar un celular en la basura, en lugar de llevarlo a un sitio de recolección apropiado, representa un riesgo ambiental? Como resultado, el porcentaje más alto tuvo la respuesta: Sí con un 86,7\%; seguido de Tal vez, con un 7,7\%; y No sé y No con un $2,5 \%$, respectivamente; finalmente la opción No me interesa con un porcentaje de $0,6 \%$. 


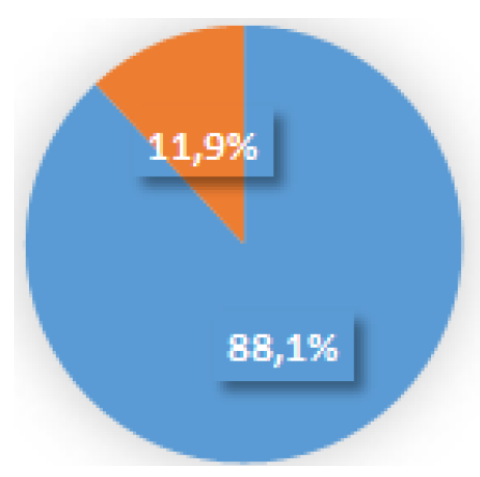

Figura 4. Conocimiento acerca de los componentes tóxicos en celulares.

Fuente: elaboración propia.

En la figura 4, se aprecian los resultados de la pregunta: ¿sabía usted que un celular tiene componentes muy tóxicos en su estructura? El 88,1 \% de los encuestados respondió Sí y el 11,9\% respondió No.

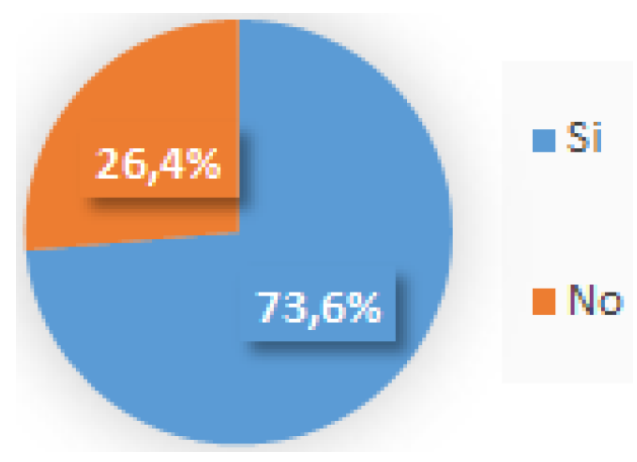

Figura 5. Conocimiento de puntos de reciclaje. Fuente: elaboración propia.

En la figura 5, se evidencian las respuestas a la pregunta: ¿tiene conocimiento de puntos que reciclen celulares que ya no utiliza? A lo que se obtuvo un $73,6 \%$ en la opción Sí, y No obtuvo un 26,4 \%.

Por otro lado, con base a determinar qué tan dispuestas están las personas a contribuir en alternativas que estén dirigidas a la promoción de tecnología verde, se realizaron algunas preguntas que se exponen a continuación:

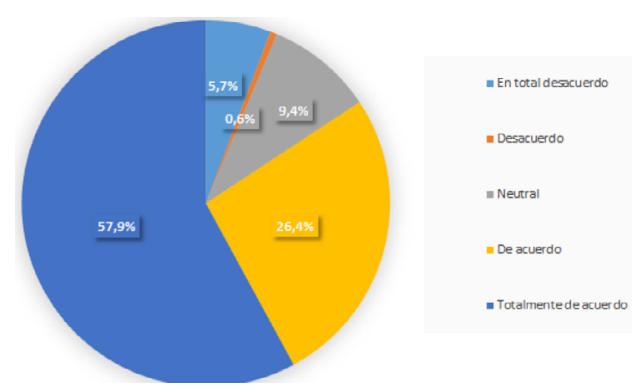

Figura 6. Disposición para participar en estrategias que ayuden al medio ambiente. Fuente: elaboración propia.

En la figura 6, se evidencian los resultados correspondientes a la pregunta: ¿qué tan de acuerdo estaría con ser parte de una estrategia enfocada al beneficio de medio ambiente por medio del adecuado reciclaje de teléfonos móviles? El 57,9 \% de la población encuestada se encuentra Totalmente e acuerdo y un $26,4 \%$ se encuentra De acuerdo en aportar en estrategias enfocadas al beneficio del medio ambiente. Por otro lado, un 9,4\% adopta una posición Neutral, mientras que 0,6 $\%$ se torna en Desacuerdo y un 5,7 \% se encuentra en Total desacuerdo en las es- 
trategias de reciclaje de teléfonos móviles para el beneficio del medio ambiente.

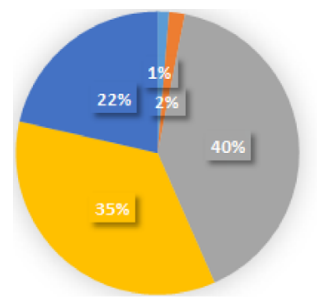

- Para Nada dispuesto

- No dispuesto

a Indeciso

a Dispuesto

- Muy dispuesto

Figura 7. Disposición de participación en una estrategia ecoamigable.

Fuente: elaboración propia.

En la figura 7, se dan a conocer los porcentajes responden a la pregunta: ¿qué tan dispuesto usted se sentiría al pagar de más por un celular ecoamigable? El 21,4 $\%$ de los encuestados se encuentra Muy dispuesto a comprarlos, un 35,2 \% se encuentra Dispuesto, un 40,3\% adopta una posición Indecisa; por otro lado, un 1,8\% No se encuentra dispuesto y un $1,3 \%$ no se encuentra Para nada dispuesto.

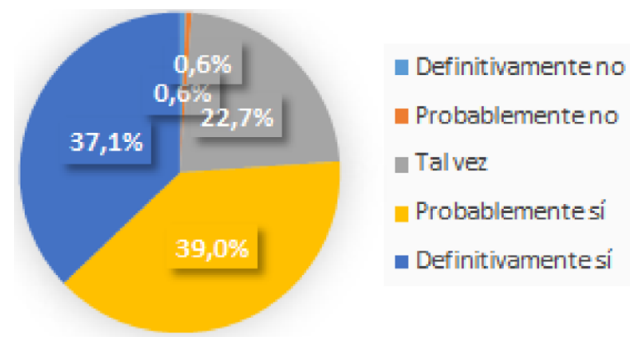

Figura 8. Recomendación a conocidos de participar en la estrategia eco-amigable. Fuente: elaboración propia.
En la figura 8, se exhiben las respuestas a la pregunta: ¿les recomendaría a personas cercanas a usted ser parte de la estrategia expuesta anteriormente? A lo que un $39 \%$ respondió que Probablemente sí, el 37,1\% respondió que Definitivamente sí; seguido a estos, un $22,7 \%$ respondió Tal vez; y, por último, las opciones Definitivamente no y Probablemente no corresponden a un $0,6 \%$, cada una.

\section{Discusión y conclusiones}

Según los resultados expuestos anteriormente, respecto a las actitudes y percepciones de la población en estudio frente al manejo de los celulares que ya no utiliza y su incidencia en el medio ambiente, se puede inferir que casi toda la población tiene conocimiento del daño que podría causar desechar un celular indebidamente, dado que las razones por las que han cambiado sus dispositivos se inclinan hacia una necesidad de adquirir uno, ya sea por lo que fue hurtado, le era insuficiente o culmino su vida útil; y no por cuestiones de estética, moda o gusto. Además, se tienen en cuenta las actitudes que toman las personas encuestadas con los celulares que ya no utilizan, las cuales son: regalar, guardar, o incluso, llevar los dispositivos a sitios especializados de re- 
colección, en vez de botarlos directamente a la basura, ya que podría causar daño al medio ambiente. En relación y soporte con lo anteriormente mencionado, gran parte del objeto en estudio tiene conocimiento de la presencia de materiales tóxicos en los celulares, pero lamentablemente es posible notar que la mayoría de las personas desecha de manera inadecuada el celular que ya no utilizan, debido a que solo una tercera parte conocen de sitios especializados de reciclaje y recolección.

Contrastando un estudio anteriormente realizado por Pascuas et al. (2018) y el presente estudio, es posible resaltar que las dos investigaciones llegan a un punto donde se evidencia que las personas encuestadas son conscientes y tienen conocimiento del daño que puede causar un dispositivo móvil desechado indebidamente; sin embargo, el desconocimiento por parte de ellas de los puntos de recolección adecuados para estos dispositivos, contribuye a que estas actúen de una forma contraria, afectando el medio ambiente.

Analizando a fondo la percepción general de los encuestados, y qué tan de acuerdo se sienten con esta solución de cambio para mitigar dicha problemática ambiental (figura 6), se pudo obtener que la gran mayoría manifiesta estar confor- me con la solución y, además, se apunta al cambio. Aunque, por otra parte, el restante del porcentaje de los encuestados manifiesta sentirse en desacuerdo o en una posición completamente neutral. No obstante, con el propósito de tratar temas un poco más de rentabilidad y monetarios, se obtuvieron resultados ante la disposición económica del consumidor en general, si este quisiese adquirir un dispositivo ecoamigable. Más de la mitad de los encuestados manifestaron estar dispuestos a invertir más en un dispositivo que ayude con el medio ambiente, aunque, por otro lado, el resto de votantes optaron por una posición indecisa o rotundamente negativa. Con base en todo lo anterior y teniendo en cuenta que la mayoría de las propuestas tuvieron una excelente respuesta ante los encuestados, la última pregunta fue enfocada en la disposición de divulgación del producto, dependiendo de su funcionamiento, es decir, si el consumidor llega a tener una grata experiencia con los dispositivos, qué tan comprometido y dispuesto estaría de recomendar dicho dispositivo.

El estudio "What 's next: eco friendly cellphones made using green materials" (EcoFriend, 2011) refiere que este tipo de dispositivos ecoamigables no son un invento nada antiguo, por el contrario, tienen cierto grado de innova- 
ción actual, $\mathrm{y}$ tanto sus funciones como su estructura interna son algo desconocidas por los compradores. Existen varios factores en pro y contra a la hora de considerar este drástico cambio a ecocelulares; no obstante, la primera acogida de estos dispositivos nuevos no fue muy satisfactoria, pero, sin duda alguna, hizo parte de un gran impulso y abrebocas para lo que se viene en el futuro.

De lo anteriormente expuesto, se infiere la relevancia de masificar la información sobre el reciclaje y el adecuado desecho de los dispositivos electrónicos, ya que estas son problemáticas que causan un daño enorme hacia el medio ambiente y los perjudicados serán las futuras generaciones. Como se pudo observar en el estudio, la mayoría de personas ven esto como un tema reiterativo, y que a la vez les genera desinterés, puesto que no se les brinda la información específica y necesaria, la cual, les pueda dar otra perspectiva y así lograr que las personas no hagan caso omiso al tema, y se ayude a mitigar el daño medioambiental.

Los datos obtenidos de esta investigación pueden servir como información relevante para los fabricantes de teléfonos al momento de la producción de los mismos, para así reducir el uso de metales tóxicos y su impacto negativo a futuro.

\section{Referencias}

Christensen, P. H. \& Foss, N. J. (2021). Present-but-online: How mobile devices may harm purposeful co-presence in organizations (and what can be done about it). European Management Journal, 39(1), 84-94. https://doi.org/10.1016/j. emj.2020.07.006

Coyne, S. M., Stockdale, L. \& Summers, K. (2019). Problematic cell phone use, depression, anxiety, and self-regulation: Evidence from a three year longitudinal study from adolescence to emerging adulthood. Computers in Human Behavior, (96), 78-84. https://doi.org/10.1016/j. chb.2019.02.014

Dang, D. H. \& Zhang, Z. (2021). Hazardous motherboards: Changes in metal contamination related to the evolution of electronictechnologies. Environmental Pollution, (268), 115731. https://doi.org/10.1016/j. envpol.2020.115731

Deen, T. (2019, 25 de enero). El mundo tiene más basura electrónica que humanos. Inter Press Service. https://ipsnoticias. net/2019/01/mundo-mas-basura-electronica-humanos/

EcoFriend. (2011). What's Next: Eco friendly cell phones made using green materials. https://ecofriend.com/eco-friendly-cellphones-green-materials.html

Fujii, H. \& Managi, S. (2019). Decomposition analysis of sustainable green technology inventions in China. Technological Forecasting and Social Change, (139), 10-16. https://doi.org/https://doi.org/10.1016/j.techfore.2018.11.013 
Mihai, F. C., Gnoni, M. G., Meidiana, C., Ezeah, C. \& Elia, V. (2019). Chapter 1 Waste Electrical and Electronic Equipment (WEEE): Flows, Quantities, and Management-A Global Scenario. En M. N. Vara Prasad \& M. Vithanage (eds.), Electronic Waste Management and Treatment Technology (pp. 1-34). Butterworth-Heinemann. https://doi.org/10.1016/B978-0-12816190-6.00001-7

Nnorom, I. C., Ohakwe, J. \& Osibanjo, O. (2009). Survey of willingness of residents to participate in electronic waste recycling in Nigeria - A case study of mobile phone recycling. Journal of Cleaner Production, 17(18), 1629-1637. https://doi.org/10.1016/j.jclepro.2009.08.009

Pascuas, Y., Chico, D. y Hernández, S. (2018). Residuos de celulares y tabletas: incidencia del nivel de escolaridad en las actitudes y percepciones frente a su manejo. Revistya U.D.C.A Actualidad \& Divulgación Cientifica, 21(1), 243-252. https:// revistas.udca.edu.co/index.php/ruadc/article/view/683
Ruiz Galeano, D. A. \& Bautista Rodríguez, S. C. (2021). An integrated method of environmental analysis and system dynamics for management of mobile phone waste in Colombia. Journal of Cleaner Production, (279), 123768. https://doi.org/10.1016/j. jclepro.2020.123768

Singh, N., Duan, H., Ogunseitan, O. \& Tang, Y. (2019). Toxicity trends in E-Waste: A comparative analysis of metals in discarded mobile phones. Journal of Hazardous Materials, (380), 120898. https://doi.org/10.1016/j.jhazmat.2019.120898

Zornosa Prieto, H. E. (2009). El riesgo asegurable y los riesgos emergentes de las nuevas tecnologías. Revista de Derecho Privado, (17), 141-73. https://revistas.uexternado. edu.co/index.php/derpri/article/view/413 